BOOSTER POLARITY STANDARDS

BOOSTER TECHNICAL NOTE

NO. 180

E. BLESER

OCTOBER 30, 1990

ALTERNATING GRADIENT SYNCHROTRON DEPARTMENT BROOKHAVEN NATIONAL LABORATORY UPTON, NEW YORK 11973 


\section{BOOSTER POLARITY STANDARDS}

A. Booster Magnet Polarity Standard

1. The EP\&S has a magnet polarity standard that they have been following for twenty years. To the best of its abilities the Booster shall follow that standard. The EP\&S standard is summarized in Figure 1 and in paragraph 3.

2. Figure 1 (prepared by C. Pearson in consultation with W. Glenn) shows the EP\&S convention. Looking along the beam line, magnets with the polarities shown in the figure are in "A POLARITY". The other case is called "B POLARITY" and has the north and south poles interchanged.

3. A written definition of the standard is:

a. A "magnet" is a magnet that has no field components along the horizontal midplane, i.e., $B_{x}(x, 0)=0$. Typical "magnets" are shown in the left hand column of Figure 1.

b. A "skew magnet" is achieved by rotating a "magnet" to the right (clockwise), when looking along the beam direction, through an angle equal to one half of the angle between two adjacent poles. The result is $B_{y}(0, y)=0$. Typical "skew magnets" are shown in the right hand column of Figure 1. In this terminology a pitching dipole is a skew dipole.

c. When looking along the beam direction through a "magnet", "A POLARITY" is defined as that polarity for which the right hand pair of poles has the North pole above the South pole. It follows from this definition that a "skew magnet" has a North pole on the midplane on the right hand side.

d. "B POLARITY" has the North and South poles interchanged.

e. Any other situations are special cases and must be treated as such.

4. Application of the Standard

a. Each magnet as it is manufactured is labeled in a uniform way. The labelling designates:

(i) One of the magnet leads as the "T" lead. 


\section{AGS Magnet Polarity Standards}

The following magnets are presented in " $A$ ". polarity assuming that the beam is traveling into the paper.

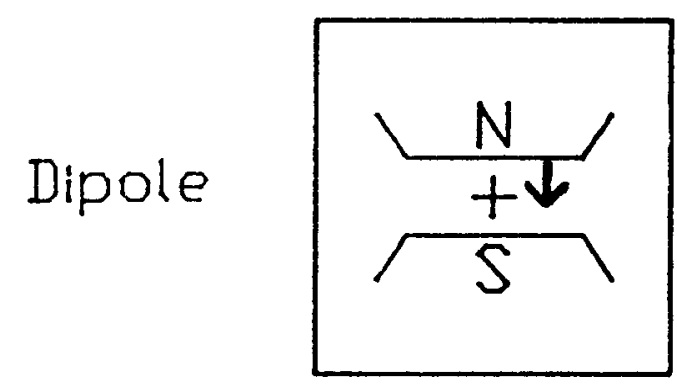

Pitching

Dipole

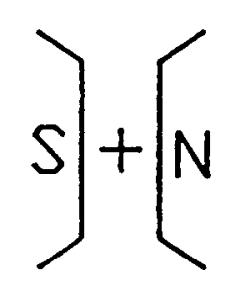

Quadrupole $\frac{N}{S+(N)}$

Skew

Quadrupole

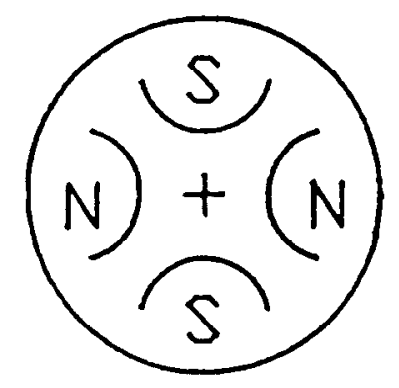

Sextupole $\left(\begin{array}{l}\left.\frac{N}{N}\right)^{\frac{N}{S}}+\frac{(N}{(N} \\ S\end{array}\right.$

Skew
Sextupole
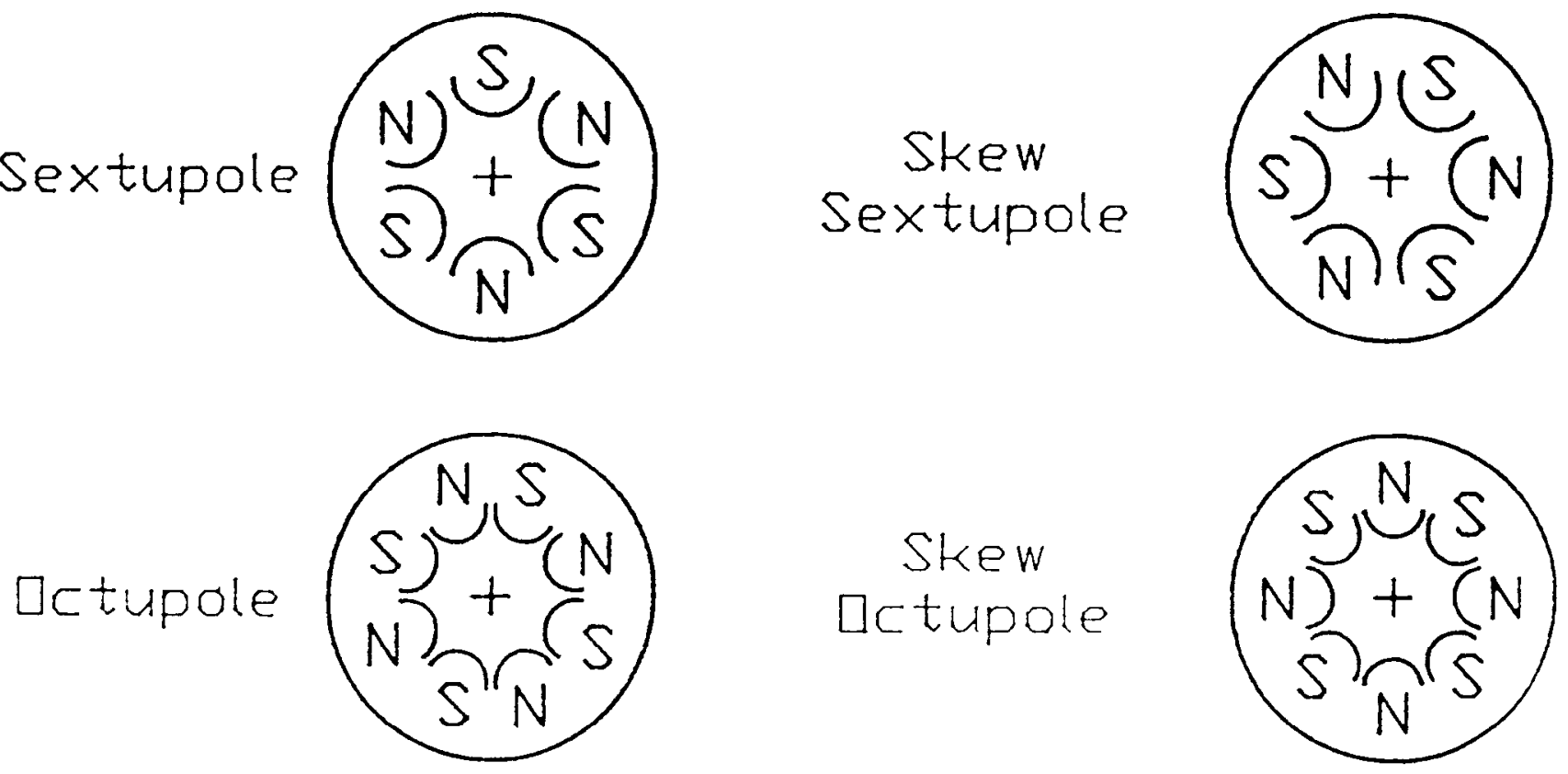
(ii) A beam direction through the magnet must be designated for those magnets for which rotation through $180^{\circ}$ about the vertical axis produces a change in polarity. Those magnets for which a beam direction must be specified are: quadrupoles, octupoles, etc., skew dipoles, skew sextupoles, etc. Those magnets for which a beam direction need not be specified are: dipoles, sextupoles, etc., skew quadrupoles, skew octupoles, etc.

(iii) If confusion is possible a top and bottom or "up" direction should be designated.

Then the standard is that:

POSITIVE CURRENT INTO THE "T" LEAD PRODUCES

"A POLARITY"

b. The beam designer is now responsible for designating each magnet in his beam line as being in either "A" or "B" polarity.

c. The cable runs must now be designed so that the "T" lead of an "A" magnet is connected to the positive output of a power supply, and so forth. Appendix II deals with special cases.

5. A single steel core may have several independently powered sets of coils - a main coil and one or more sets of trim coils. Each set of coils is viewed as a magnet for the purposes of this note.

B. Booster Power Supply Polarity Standard

1. Monopolar Supply

a. The monopolar supplies are very simple. They have two terminals on the back, labelled plus and minus by the manufacturer. The plus terminal is a source of positive current.

2. Bipolar Supply

a. The bipolar supplies are more complicated. They have two terminals on the back. We shall label these terminals "1" and "2". This choice is based on a desire to avoid confusion with " +" and "-" or "A" and "B" which are already in use. The labelling shall be carried out so 
that when a positive signal is given to the power supply it will put out positive current at terminal "1".

b. By convention the polarity of a magnet connected to a bipolar supply shall be designated as the polarity that it has when positive current is coming out of terminal " 1 ".

c. Switches of various sorts do not seem to be pertinent to the Booster at the present time.

\section{OBSERVATIONS on BOOSTER POLARITY STANDARDS}

The standards given above are intended to be complete and unambiguous. They seem to achieve these goals and to be generally acceptable. This note, therefore, marks their official promulgation. Any complications or insufficiencies should be brought to the author's attention.

The remainder of this section notes certain results that follow from the above and certain mnemonics that may be useful.

A. Dipoles

1. The main "dipoles" in the AGS are "A" polarity. The main dipoles in the Booster are "B" polarity.

B. Quadrupoles

1. In the standard jargon, a "focussing" or "horizontal" quadrupole is a horizontally focussing quadrupole, and a "defocussing" or "vertical" quadrupole is a horizontally defocussing quadrupole.

2. For a positive beam a "focussing" or "horizontal" quadrupole is " $A$ " polarity. A "defocussing" or "vertical" quadrupole is "B" polarity.

3. In a beam line paragraph 1 applies in a clear cut way. In an accelerator a horizontal quad is normally at a maximum in the horizontal beta function. A vertical quad is normally at a maximum in the vertical beta function. Other elements at these locations are sometimes designated "horizontal" or "vertical" because of their location rather than because of their function. No confusion should arise. 
4. In the Booster there are complicated families of half integer stop band correctors and skew quads. Their polarities have been assigned consistent with Gardner's requirements.

\section{Sextupoles}

1. Horizontal sextupoles are located at horizontal beta maximum and control principally the horizontal chromaticity. Vertical sextupoles are located at vertical beta maximum and control principally the vertical chromaticity.

2. To increase the horizontal chromaticity the horizontal string should be in "B" polarity, and to increase the vertical chromaticity the vertical string in " $\mathrm{A}$ " polarity. These polarities are opposite to the polarities of the adjacent quadrupoles.

3. The third integer stop band families are in accord with Gardner.

\section{RECOMMENDED CONVENTIONS}

The only important ambiguities occur when we are dealing with bipolar trim and correction magnets. These ambiguities are not of great practical consequence since they can be resolved by changing a sign in the computer program or by switching leads as appropriate. However, since the hardest thing to get right is the sign, it is desirable to establish some clear cut general principles to guide the designer and to make life easier for the installer and user.

The recommended Booster convention is:

The general convention for assigning polarities shall be that in cases where there is an option, i.e., for most bipolar supplies, the system shall be hooked up in "A" polarity. For bipolar magnets " $A$ " polarity is defined in paragraph I.B.2.b, above.

\section{OBSERVATIONS ON BOOSTER POLARITY CONVENTIONS}

A. In An Accelerator

It is nice in an accelerator if positive current in a secondary (not main) horizontal dipole type magnet kicks the beam to a larger radius (to the outside of the machine).

1. Note that in the AGS where the main "dipoles" are "A" polarity, the secondary dipoles are largely "B" polarity, and thus follow the above precept. 
This came about because the AGS convention at the time was to select "B" polarity when there was a choice.

2. In the Booster where the main dipoles are "B" polarity the secondary dipoles will be largely " $A$ " polarity by the convention in Part III above. This fortunately also agrees with the precept above.

3. Bipolar skew or pitching dipoles should all be "A" polarity.

\section{B. In A Beam Line}

1. The beam line designer should designate the polarity of the main magnets.

2. We expect that all the main magnets will be monopolar.

3. Independent bipolar correction magnets in a beam line should follow the convention given in III.

\section{CHECK-OUT CONVENTIONS}

The development of these standards has been very complex since numerous possibilities have been suggested and strongly argued for. The situation is exactly described by the story of the seven Indian fakirs assigned to describe an elephant. Although they were very wise they were also very blind and as we all know each described the elephant from his point of view. So for our Booster. No simple conventions seemed generally useful from all points of view. This is of no particular consequence as long as we achieve our goals of full and complete documentation. The principal means of documentation will be the Booster Data Base, which will include complete documentation on all the wiring lists and on all the polarity assignments. This information will be available as "walking lists" for the detailed check-out of the various systems. The Booster is complex enough so that hardware checking should be done from documentation, not from memory nor from rules of thumb.

From the operators' point of view, the control system will contain two pages, a device page and an operating page. The device page will be coupled to the power supplies in the most transparent straightforward way possible - a positive signal at the device page producing a positive signal at the power supply input. Between the device page and the operating page there may exist sign inversions for many devices, the goal being to make the operating page user friendly. Thus the system we have adopted is to make the hook-up and check-out of the electrical system straightforward and easy but based entirely on complete and suitable documentation. The computer operating pages will be straightforward, easy, and user friendly from the operators' point of view. The hard task will be that of the programmer who will have to make the translation from the device page to the operating 
page. The coordinating physicists will have to work very closely with the programmers to be sure that the correct polarity translations are made. 


\section{Appendix I}

This appendix lists the assigned polarities of all the magnets for the Booster, the Linac to Booster line and the Booster to AGS line. 
LTB LINE POLARITIES

\begin{tabular}{|c|c|c|c|c|}
\hline MAGNETS & NO. & POLARITY & P.S. & TYPE \\
\hline DH1 & 1 & B & 1 & MONO \\
\hline DH2,DH3,DH4,DH5 & 4 & B & 1 & MONO \\
\hline QH1 & 1 & B & 1 & MONO \\
\hline QH3 & 1 & B & 1 & MONO \\
\hline QH5 & 1 & B & 1 & MONO \\
\hline QH6 & 1 & B & 1 & MONO \\
\hline QH7 & 1 & B & 1 & MONO \\
\hline QH8 & 1 & B & 1 & MONO \\
\hline QH10 & 1 & B & 1 & MONO \\
\hline QH12 & 1 & B & 1 & \\
\hline QV2 & 1 & $\mathbf{A}$ & 1 & MONO \\
\hline QV4 & 1 & $\mathbf{A}$ & 1 & MONO \\
\hline QV9 & 1 & $\mathbf{A}$ & 1 & MONO \\
\hline QV11 & 1 & $\mathbf{A}$ & 1 & MONO \\
\hline QV13 & 1 & $\mathbf{A}$ & 1 & MONO \\
\hline DH015 & 1 & $\mathbf{A}$ & 1 & BIP \\
\hline DH076 & 1 & $\mathbf{A}$ & 1 & BIP \\
\hline DH088 & 1 & $\mathbf{A}$ & 1 & BIP \\
\hline DV018 & 1 & $\mathbf{A}$ & 1 & BIP \\
\hline DV026 & 1 & A & 1 & BIP \\
\hline DV082 & 1 & $\mathbf{A}$ & 1 & BIP \\
\hline DV095 & 1 & $\mathbf{A}$ & 1 & BIP \\
\hline DV112 & 1 & $\mathbf{A}$ & 1 & MONO \\
\hline
\end{tabular}


BTA LINE POLARITIES

\begin{tabular}{|c|c|c|c|c|}
\hline MAGNETS & No. & POLARITY & P.S. & TYPE \\
\hline DH1 & 1 & B & 1 & MONO \\
\hline DH2, DH3 & 2 & B & 1 & MONO \\
\hline DH4 & 1 & B & 1 & MONO \\
\hline DH5 & 1 & A & 1 & MONO \\
\hline QV1 & 1 & B & 1 & MONO \\
\hline QV3 & 1 & B & 1 & MONO \\
\hline QV5 & 1 & B & 1 & MONO \\
\hline QV7 & 1 & B & 1 & MONO \\
\hline QV9 & 1 & B & 1 & MONO \\
\hline QV11 & 1 & B & 1 & MONO \\
\hline QV13 & 1 & B & 1 & MONO \\
\hline QV15 & 1 & B & 1 & MONO \\
\hline QH2A-QH2B & 2 & $\mathbf{A}$ & 1 & MONO \\
\hline QH4 & 1 & $\mathbf{A}$ & 1 & MONO \\
\hline QH6 & 1 & $\mathbf{A}$ & 1 & MONO \\
\hline QH8 & 1 & $\mathbf{A}$ & 1 & MONO \\
\hline QH10 & 1 & $\mathbf{A}$ & 1 & MONO \\
\hline QH12 & 1 & $\mathbf{A}$ & 1 & MONO \\
\hline QH14 & 1 & $\mathbf{A}$ & 1 & MONO \\
\hline DV007 & 1 & $\mathbf{A}$ & 1 & BIP \\
\hline DV141 & 1 & $\mathbf{A}$ & 1 & BIP \\
\hline DV168 & 1 & $\mathbf{A}$ & 1 & BIP \\
\hline DV181 & 1 & $\mathbf{A}$ & 1 & BIP \\
\hline DH127 & 1 & $\mathbf{A}$ & 1 & BIP \\
\hline DH158 & 1 & $\mathbf{A}$ & 1 & BIP \\
\hline SPTM F6 & 1 & $\mathbf{A}$ & 1 & MONO \\
\hline SPTM L20 & 1 & B & 1 & MONO \\
\hline
\end{tabular}


BOOSTER MAGNET POLARITIES

\begin{tabular}{|c|c|c|c|c|c|}
\hline MAGNETS & No. & POLARITY & P.S. & TYPE & $\begin{array}{l}\text { MAGNET } \\
\text { LOCATION }\end{array}$ \\
\hline \multicolumn{6}{|l|}{ Main Magnets } \\
\hline Main Magnet String & & & 1 & MONO & \\
\hline Dipoles $\quad$ DH & 36 & B & & & \\
\hline Hor Quads QH & 24 & $\mathbf{A}$ & & & EVEN \\
\hline Vert Quads QV & 24 & B & & & ODD \\
\hline Hor Sext Str SH & 24 & B & 1 & MONO & EVEN \\
\hline Vert Sext Str SV & 24 & $\mathbf{A}$ & 1 & MONO & ODD \\
\hline \multicolumn{6}{|l|}{ Correction Magnets } \\
\hline Hor Dipoles DHC & 24 & $\mathbf{A}$ & 24 & BIP & EVEN \\
\hline Vert Dipoles DVC & 24 & $\mathbf{A}$ & 24 & BIP & ODD \\
\hline \multicolumn{6}{|l|}{ Skew Quads QSC } \\
\hline QS-STR1 & 6 & & 1 & BIP & ODD \\
\hline QSCE1 & & A & & & \\
\hline QSCE7 & & $\mathbf{A}$ & & & \\
\hline QSCA1 & & $\mathbf{A}$ & & & \\
\hline QSCA7 & & $\mathbf{A}$ & & & \\
\hline QSCC1 & & $\mathbf{A}$ & & & \\
\hline QSCC7 & & $\mathbf{A}$ & & & \\
\hline & & & & & \\
\hline QS-STR2 & 6 & & 1 & BIP & EVEN \\
\hline QSCE2 & & $\mathbf{A}$ & & & \\
\hline QSCE8 & & $\mathbf{A}$ & & & \\
\hline QSCA2 & & $\underline{A}$ & & & \\
\hline QSCA8 & & $\mathbf{A}$ & & & \\
\hline QSCC2 & & $\mathbf{A}$ & & & \\
\hline QSCC8 & & $\mathbf{A}$ & & & \\
\hline OS-STR3 & 6 & & 1 & BIP & ODD \\
\hline QSCD7 & & A & & & \\
\hline QSCF1 & & $\mathbf{A}$ & & & \\
\hline QSCF7 & & $\mathbf{A}$ & & & \\
\hline QSCB1 & & $\mathbf{A}$ & & & \\
\hline QSCB7 & & $\mathbf{A}$ & & & \\
\hline
\end{tabular}




\begin{tabular}{|c|c|c|c|c|c|}
\hline MAGNETS & No. & POLARITY & P.S. & TYPE & $\begin{array}{l}\text { MAGNET } \\
\text { LOCATION }\end{array}$ \\
\hline QSCD1 & & $\mathbf{A}$ & & & \\
\hline QS-STR4 & 6 & & 1 & BIP & EVEN \\
\hline QSCD8 & & $\mathbf{A}$ & & & \\
\hline QSCF2 & & A & & & \\
\hline QSCF8 & & $\mathbf{A}$ & & & \\
\hline QSCB2 & & $\mathbf{A}$ & & & \\
\hline QSCB8 & & $\mathbf{A}$ & & & \\
\hline QSCD2 & & $\mathbf{A}$ & & & \\
\hline \multicolumn{6}{|c|}{ Dipole Trim Magnets } \\
\hline \multicolumn{6}{|c|}{ Proton Inject. Bump } \\
\hline TDHC4 & 1 & $\mathbf{A}$ & 1 & MONO & \\
\hline TDHC8 & 1 & $\mathbf{A}$ & 1 & MONO & \\
\hline TDHD1 & 1 & $\mathbf{A}$ & 1 & MONO & \\
\hline \multicolumn{6}{|c|}{ HI Injection Bump } \\
\hline TDHB8 & 1 & B & 1 & MONO & \\
\hline TDHC1 & 1 & B & 1 & MONO & \\
\hline TDHC5 & 1 & B & 1 & MONO & \\
\hline \multicolumn{6}{|l|}{ Extraction Bump } \\
\hline TDHF2 & 1 & $\mathbf{A}$ & 1 & MONO & \\
\hline TDHF4 & 1 & $\underline{A}$ & 1 & MONO & \\
\hline TDHF7 & 1 & $\mathbf{A}$ & 1 & MONO & \\
\hline TDHA1 & 1 & $\mathbf{A}$ & 1 & MONO & \\
\hline \multicolumn{6}{|l|}{ Dump Bump } \\
\hline TDHD2 & 1 & B & 1 & MONO & \\
\hline TDHD4 & 1 & B & 1 & MONO & \\
\hline TDHD7 & 1 & B & 1 & MONO & \\
\hline TDHE1 & 1 & B & 1 & MONO & \\
\hline
\end{tabular}




\begin{tabular}{|c|c|c|c|c|c|}
\hline MAGNETS & No. & POLARITY & P.S. & TYPE & IMAGNFT \\
\hline \multicolumn{6}{|l|}{ Quad Trim Magnets } \\
\hline Hor Tune Str TUQH & 24 & $\mathbf{A}$ & 1 & BIP & EVEN \\
\hline Vert Tune Str TUQV & 24 & $\mathbf{A}$ & 1 & BIP & ODD \\
\hline \multicolumn{6}{|l|}{$1 / 2$ Integer Stop Bands } \\
\hline QV-STR1 & & & 1 & BIP & ODD \\
\hline TQVD7 & 1 & B & & & \\
\hline TQVE1 & 1 & $\mathbf{A}$ & & & \\
\hline TQVE7 & 1 & $\mathbf{A}$ & & & \\
\hline TQVF1 & 1 & B & & & \\
\hline TQVF7 & 1 & B & & & \\
\hline TQVA1 & 1 & $\mathbf{A}$ & & & \\
\hline TQVA7 & 1 & $\mathbf{A}$ & & & \\
\hline TQVB1 & 1 & B & & & \\
\hline TQVB7 & 1 & B & & & \\
\hline TQVC1 & 1 & $\mathbf{A}$ & & & \\
\hline TQVC7 & 1 & $\mathbf{A}$ & & & \\
\hline TQVD1 & 1 & B & & & \\
\hline \\
\hline QH-STR1 & & & 1 & BIP & EVEN \\
\hline TQHD8 & 1 & B & & & \\
\hline TQHE2 & 1 & $\mathbf{A}$ & & & \\
\hline TQHE8 & 1 & $\mathbf{A}$ & & & \\
\hline TQHF2 & 1 & B & & & \\
\hline TQHF8 & 1 & B & & & \\
\hline TQHA2 & 1 & $\mathbf{A}$ & & & \\
\hline TQHA8 & 1 & $\mathbf{A}$ & & & \\
\hline TQHB2 & 1 & B & & & \\
\hline TQHB8 & 1 & B & & & \\
\hline TQHC2 & 1 & $\mathbf{A}$ & & & \\
\hline TQHC8 & 1 & $\mathbf{A}$ & & & \\
\hline TQHD2 & 1 & B & & & \\
\hline OV-STR2 & & & 1 & BIP & ODD \\
\hline TQVD5 & 1 & $\mathbf{A}$ & & & \\
\hline
\end{tabular}




\begin{tabular}{|c|c|c|c|c|c|}
\hline MAGNETS & NO. & POLARITY & P.S. & TYPE & $\begin{array}{l}\text { MAGNET } \\
\text { LOCATION }\end{array}$ \\
\hline TQVE3 & 1 & $\mathbf{A}$ & & & \\
\hline TQVE5 & 1 & B & & & \\
\hline TQVF3 & 1 & B & & & \\
\hline TQVF5 & 1 & $\mathbf{A}$ & & & \\
\hline TQVA3 & 1 & $\mathbf{A}$ & & & \\
\hline TQVA5 & 1 & B & & & \\
\hline TQVB3 & 1 & B & & & \\
\hline TQVB5 & 1 & $\mathbf{A}$ & & & \\
\hline TQVC3 & 1 & $\mathbf{A}$ & & & \\
\hline TQVC5 & 1 & B & & & \\
\hline TQVD3 & 1 & B & & & \\
\hline QH-STR2 & & & 1 & BIP & EVEN \\
\hline TQHD4 & 1 & B & & & \\
\hline TQHD6 & 1 & $\mathbf{A}$ & & & \\
\hline TQHE4 & 1 & $\mathbf{A}$ & & & \\
\hline TQHE6 & 1 & B & & & \\
\hline TQIIF4 & 1 & B & & & \\
\hline TQHF6 & 1 & $\mathbf{A}$ & & & \\
\hline TQHA4 & 1 & $\underline{A}$ & & & \\
\hline TQHA6 & 1 & B & & & \\
\hline TQHB4 & 1 & B & & & \\
\hline TQHB6 & 1 & $\mathbf{A}$ & & & \\
\hline TQHC4 & 1 & $\mathbf{A}$ & & & \\
\hline TQHC6 & 1 & B & & & \\
\hline \multicolumn{6}{|c|}{ Sext. Trim Magnets } \\
\hline \multicolumn{6}{|c|}{$1 / 3$ Integer Stop Band } \\
\hline SV-STR1 & & & 1 & BIP & ODD \\
\hline TSVE1 & $1 / 2$ & B & & & \\
\hline TSVE3 & $1 / 2$ & $\mathbf{A}$ & & & \\
\hline TSVA1 & 1 & $\mathbf{A}$ & & & \\
\hline TSVA3 & 1 & B & & & \\
\hline TSVC1 & $1 / 2$ & B & & & \\
\hline TSVC3 & $1 / 2$ & $\mathbf{A}$ & & & \\
\hline
\end{tabular}




\begin{tabular}{|c|c|c|c|c|c|}
\hline MAGNETS & No. & POLARITY & P.S. & TYPE & MAGNFT \\
\hline SII-STR1 & & & 1 & BIP & EVEN \\
\hline TSHE2 & $1 / 2$ & B & & & \\
\hline TSHEA & $1 / 2$ & $\mathbf{A}$ & & & \\
\hline TSHA2 & 1 & $\mathbf{A}$ & & & \\
\hline TSHA4 & 1 & B & & & \\
\hline TSHC2 & $1 / 2$ & B & & & \\
\hline TSHC4 & $1 / 2$ & $\mathbf{A}$ & & & \\
\hline & & & & & \\
\hline SV-STR2 & & & 1 & BIP & ODD \\
\hline TSVE5 & $1 / 2$ & B & & & \\
\hline TSVE7 & $1 / 2$ & $\mathbf{A}$ & & & \\
\hline TSVA5 & 1 & $\mathbf{A}$ & & & \\
\hline TSVA7 & 1 & B & & & \\
\hline TSVC5 & $1 / 2$ & B & & & \\
\hline TSVC7 & $1 / 2$ & $\mathbf{A}$ & & & \\
\hline & & & & & \\
\hline SH-STR2 & & & 1 & BIP & EVEN \\
\hline TSHE6 & $1 / 2$ & B & & & \\
\hline TSHE 8 & $1 / 2$ & $\mathbf{A}$ & & & \\
\hline TSHA6 & 1 & $\mathbf{A}$ & & & \\
\hline TSHA8 & 1 & B & & & \\
\hline TSHC6 & $1 / 2$ & B & & & \\
\hline TSHC8 & $1 / 2$ & $\mathbf{A}$ & & & \\
\hline & & & & & \\
\hline SV-STR3 & & & 1 & BIP & ODD \\
\hline TSVF1 & $1 / 2$ & B & & & \\
\hline TSVF3 & $1 / 2$ & A & & & \\
\hline TSVB1 & $1 / 2$ & B & & & \\
\hline TSVB3 & $1 / 2$ & $\mathbf{A}$ & & & \\
\hline TSVD1 & 1 & $\mathbf{A}$ & & & \\
\hline TSVD3 & 1 & B & & & \\
\hline & & & & & \\
\hline SH-STR3 & & & 1 & BIP & EVEN \\
\hline TSHF2 & $1 / 2$ & B & & & \\
\hline TSHF4 & $1 / 2$ & $\mathbf{A}$ & & & \\
\hline TSHB2 & $1 / 2$ & B & & & \\
\hline
\end{tabular}




\begin{tabular}{|c|c|c|c|c|c|}
\hline MAGNETS & NO. & POLARITY & P.S. & TYPE & $\begin{array}{l}\text { MAGNET } \\
\text { LOCATION }\end{array}$ \\
\hline TSHB4 & $1 / 2$ & $\mathbf{A}$ & & & \\
\hline TSHD2 & 1 & $\mathbf{A}$ & & & \\
\hline TSHD4 & 1 & B & & & \\
\hline SV-STR47 & & & 1 & BIP & ODD \\
\hline TSVD5 & 1 & $\mathbf{A}$ & & & \\
\hline TSVD7 & 1 & B & & & \\
\hline TSVF5 & $1 / 2$ & B & & & \\
\hline TSVF7 & $1 / 2$ & $\mathbf{A}$ & & & \\
\hline TSVB5 & $1 / 2$ & B & & & \\
\hline TSVB7 & $1 / 2$ & A & & & \\
\hline SH-STR4 & & & 1 & BIP & EVEN \\
\hline TSHD6 & 1 & $\mathbf{A}$ & & & \\
\hline TSHD8 & 1 & B & & & \\
\hline TSHF6 & $1 / 2$ & B & & & \\
\hline TSHF8 & $1 / 2$ & $\mathbf{A}$ & & & \\
\hline TSHB6 & $1 / 2$ & B & & & \\
\hline TSHB8 & $1 / 2$ & $\underline{\mathbf{A}}$ & & & \\
\hline \multicolumn{6}{|l|}{ Kicker Magnets } \\
\hline \multicolumn{6}{|l|}{ Heavy Ion Injection } \\
\hline KDHC1 & 1 & $\underline{\mathbf{A}}$ & 1 & MONO & \\
\hline KDHC3 & 1 & $\mathbf{A}$ & 1 & MONO & \\
\hline KDHC6 & 1 & $\mathbf{A}$ & 1 & MONO & \\
\hline \multicolumn{6}{|l|}{ Proton Injection } \\
\hline KDHC3 & 1 & B & 1 & MONO & \\
\hline KDHC6 & 1 & B & 1 & MONO & \\
\hline KDHD1 & 1 & B & 1 & MONO & \\
\hline \multicolumn{6}{|l|}{ Booster Extraction } \\
\hline KDHF3 & 1 & $\mathbf{A}$ & 1 & MONO & \\
\hline \multicolumn{6}{|l|}{ Booster Dump } \\
\hline KDH DUMP.D3 & 1 & B & 1 & MONO & \\
\hline
\end{tabular}




\begin{tabular}{|c|c|c|c|c|c|}
\hline MAGNETS & No. & POLARITYY & P.S. & TYPE & $\begin{array}{l}\text { MAGNET } \\
\text { LOCATION }\end{array}$ \\
\hline \multicolumn{6}{|l|}{ Booster Damper } \\
\hline KDH DAMP.E3 & 1 & $\mathbf{A}$ & 1 & BIP RF & \\
\hline KDV DAMP.E3 & 1 & $\underline{\mathbf{A}}$ & 1 & BIP RF & \\
\hline \multicolumn{6}{|l|}{ Booster Tune Meter } \\
\hline KDV TUNE.E3 & 1 & $\mathbf{A}$ & 1 & MONO & \\
\hline KDH TUNE.E3 & 1 & $\mathbf{A}$ & 1 & MONO & \\
\hline \multicolumn{6}{|l|}{ Septum Magnets } \\
\hline \multicolumn{6}{|l|}{ Booster Injection } \\
\hline SPTMC3 & 1 & $\mathbf{A}$ & 1 & MONO ES & \\
\hline \multicolumn{6}{|l|}{ Booster Extraction } \\
\hline SPTMF6 & 1 & $\mathbf{A}$ & 1 & MONO & \\
\hline
\end{tabular}




\section{Appendix II}

There are three different groups involved in setting the magnet polarity:

1. The accelerator theorist or beam line designer who assigns to each magnet in his system a polarity, either "A" or "B".

2. The factory which marks permanently and irrevocably on each magnet a beam direction, a magnet top and bottom, and a "T" terminal, based on the standards given in this note.

3. The wiring group which hooks up each magnet appropriately.

This appendix consists of a table intended to help the wiring group understand complex situations. 
A. When the:

Beam direction is PARALLEL to the arrow Magnet is oriented UPRIGHT

Then to achieve the designated polarity for the designated magnet, connect the POSITIVE lead to the terminal marked " $T$ " or to the unmarked terminal " 0 " as indicated. (The magnet is in its normal orientation.)

\begin{tabular}{||l|c|c||l|c|c||}
\hline \multicolumn{3}{|c||}{ A POLARITY } & \multicolumn{3}{c||}{ B POLARITY } \\
\hline \multicolumn{1}{|c|}{ TYPE } & MAGNET & SKEW & TYPE & MAGNET & SKEW \\
\hline Dipole & $\mathbf{T}$ & $\mathbf{T}$ & Dipole & 0 & 0 \\
\hline Quadrupole & $\mathbf{T}$ & $\mathbf{T}$ & Quadrupole & 0 & 0 \\
\hline Sextupole & $\mathbf{T}$ & $\mathbf{T}$ & Sextupole & 0 & 0 \\
\hline Octupole & $\mathbf{T}$ & $\mathbf{T}$ & Octupole & 0 & 0 \\
\hline Decupole & $\mathbf{T}$ & $\mathbf{T}$ & Decupole & 0 & 0 \\
\hline
\end{tabular}

B. When the:

Beam direction is ANTIPARALLEL to the arrow Magnet is oriented UPRIGHT

Then to achieve the designated polarity for the designated magnet, connect the POSITIVE lead to the terminal marked " $T$ " or to the unmarked terminal " 0 " as indicated. (The magnet is rotated from the normal orientation by $180^{\circ}$ about the vertical axis.)

\begin{tabular}{||l|c|c||l|l|c||}
\hline \multicolumn{3}{|c|}{ A POLARITY } & \multicolumn{3}{c||}{ B POLARITY } \\
\hline \multicolumn{1}{||}{ TYPE } & MAGNET & SKEW & TYPE & MAGNET & SKEW \\
\hline Dipole & T & 0 & Dipole & 0 & T \\
\hline Quadrupole & 0 & T & Quadrupole & T & 0 \\
\hline Sextupole & T & 0 & Sextupole & 0 & T \\
\hline Octupole & 0 & T & Octupole & T & 0 \\
\hline Decupole & T & 0 & Decupole & 0 & T \\
\hline
\end{tabular}


C. When the:

Beam direction is PARALLEL to the arrow

Magnet is oriented UPSIDE DOWN

Then to achieve the designated polarity for the designated magnet, connect the POSITIVE lead to the terminal marked " $T$ " or to the unmarked terminal "( $)$ " as indicated. (The magnet is rotated from the normal orientation by $180^{\circ}$ about the beam axis.)

\begin{tabular}{||l|c|c||c|c|c||}
\hline \multicolumn{1}{|c|}{ A POLARITY } & \multicolumn{3}{c||}{ B POLARITY } \\
\hline \multicolumn{1}{|c|}{ TYPE } & MAGNET & SKEW & TYPE & MAGNET & SKEW \\
\hline Dipole & 0 & 0 & Dipole & T & T \\
\hline Quadrupole & $\mathbf{T}$ & $\mathbf{T}$ & Quadrupole & 0 & 0 \\
\hline Sextupole & 0 & 0 & Sextupole & T & T \\
\hline Octupole & $\mathbf{T}$ & $\mathbf{T}$ & Octupole & 0 & 0 \\
\hline Decupole & 0 & 0 & Decupole & T & T \\
\hline
\end{tabular}

D. When the:

Beam direction is ANTIPARALLEL to the arrow Magnet is oriented UPSIDE DOWN

Then to achieve the designated polarity for the designated magnet, connect the POSITIVE lead to the terminal marked " $T$ " or to the unmarked terminal " 0 " as indicated. (The magnet is rotated from the normal orientation by $180^{\circ}$ about the horizontal axis perpendicular to the beam axis.)

\begin{tabular}{||l|c|c||c|c|c||}
\hline \multicolumn{1}{|c|}{ A POLARITY } & \multicolumn{3}{c||}{ B POLARITY } \\
\hline TYPE & MAGNET & SKEW & TYPE & MAGNET & SKEW \\
\hline Dipole & 0 & T & Dipole & T & 0 \\
\hline Quadrupole & 0 & T & Quadrupole & T & 0 \\
\hline Sextupole & 0 & T & Sextupole & T & 0 \\
\hline Octupole & 0 & T & Octupole & T & 0 \\
\hline Decupole & 0 & T & Decupole & T & 0 \\
\hline
\end{tabular}

